Raquel Cristina Muniz de Souza, Cledir Lago, Aline da Rosa Paganini, Eduarda Nichele, Fernanda Januário Batista, Luana Dal Pont, Sabrina Furtunato de Ávila, Francielle Rosso Mazzuchello, Carla Maragno, Ivanir Prá da Silva Thomé, Luiz Gustavo Martins, Joni Marcio de Farias, Priscyla Waleska Simões, Lisiane Tuon

\title{
RELATO DE VIVÊNCIAS E EXPERIÊNCIAS NA REALIDADE DO SUS - VER VIVER SUS UNESC 2014: UM RELATO DE EXPERIÊNCIA DO MUNICÍPIO DE BALNEÁRIO RINCÃO-SC
}

\author{
REPORT OF EXPERIENCES AND EXPERIENCES IN “SUS - VER VIVER SUS \\ UNESC 2014” REALITY: A REPORT OF EXPERIENCE IN BALNEÁRIO \\ RINCÃO-SC
}

\author{
Raquel Cristina Muniz de Souza ${ }^{1}$ \\ Cledir Lago ${ }^{1}$ \\ Aline da Rosa Paganini ${ }^{2}$ \\ Eduarda Nichele ${ }^{3}$ \\ Fernanda Januário Batista ${ }^{4}$ \\ Luana Dal Pont ${ }^{5}$ \\ Sabrina Furtunato de Ávila ${ }^{6}$ \\ Francielle Rosso Mazzuchello ${ }^{7}$ \\ Carla Maragno 5 \\ Ivanir Prá da Silva Thomé ${ }^{7}$ \\ Luiz Gustavo Martins ${ }^{8}$ \\ Joni Marcio de Farias ${ }^{1,9}$ \\ Priscyla Waleska Simões ${ }^{1,9}$ \\ Lisiane Tuon ${ }^{1,9}$
}

\section{RESUMO}

\footnotetext{
${ }^{1}$ Programa de Residência multiprofissional em Atenção Básica/Saúde Coletiva. Universidade do Extremo Sul Catarinense (UNESC), Criciúma, SC, Brasil.

${ }^{2}$ Curso de Psicologia. Universidade do Extremo Sul Catarinense (UNESC), Criciúma, SC, Brasil.

${ }^{3}$ Curso de Nutrição. Universidade do Extremo Sul Catarinense (UNESC), Criciúma, SC, Brasil.

${ }^{4}$ Curso de Biomedicina. Universidade do Extremo Sul Catarinense (UNESC), Criciúma, SC, Brasil.

${ }^{5}$ Curso de Farmácia. Universidade do Extremo Sul Catarinense (UNESC), Criciúma, SC, Brasil.

${ }^{6}$ Curso de Educação Física. Universidade do Extremo Sul Catarinense (UNESC), Criciúma, SC, Brasil.

${ }^{7}$ Curso de Enfermagem. Universidade do Extremo Sul Catarinense (UNESC), Criciúma, SC, Brasil.

${ }^{8}$ Curso de Odontologia. Universidade do Extremo Sul Catarinense (UNESC), Criciúma, SC, Brasil.

9 Programa de Pós-Graduação em Saúde Coletiva (PPGSCol). Universidade do Extremo Sul Catarinense (UNESC), Criciúma, SC, Brasil.
} 
O objetivo deste trabalho foi relatar a vivência dos residentes do programa de Residência Multiprofissional em Saúde Coletiva e Saúde da Família, e dos acadêmicos de diversos cursos da área da saúde no projeto Ver Viver SUS da Universidade do Extremo Sul Catarinense no ano de 2014. Esta experiência ocorreu entre 21 a 25 de julho no município de Balneário Rincão, onde os estudantes permaneceram neste município com o intuito de vivenciar a realidade do Sistema Único de Saúde (SUS) desta cidade. Desta forma, os participantes tiveram a oportunidade de conhecer diferentes instâncias em que o SUS atua. Essa experiência possibilitou o desenvolvimento da prática, conforme a teoria adquirida na academia, e permitiu que os acadêmicos desenvolvessem atividades de educação em saúde com a população e percebessem a importância e o impacto que esse diálogo educativo exerce nos usuários. Outra dimensão do projeto observada diz respeito aos desafios e possibilidades que o SUS oferece nas práticas diárias, mediante a diversidade cultural apresentada pela população.

Palavras-chave: Educação em Saúde; Sistema Único de Saúde; Saúde Pública.

\begin{abstract}
The objective was to report the experience and residents of the Multidisciplinary Residency in Public Health and Family Health program, and academic courses in pharmacy, physical education, psychology, biomedicine and nutrition project in VerViver SUS. The experience in SUS and vivencias occurred during school recess, University of Southern Santa Catarina Far UNESC occurred between 21 to July 25 of 2014 in the city of BalnearioRincão, where residents were immersed in the city during this period. All selected students had the opportunity to meet different instances in which the SUS operates. This experience enabled the practical learning acquired not in the gym, because it allowed the students to develop health education activities with the population, and realize the importance and the impact that this educational dialogue plays in users. Another dimension of the project observed with regard to the challenges and possibilities that the SUS provides in daily practices through cultural diversity of populations.

Keywords: Health education; Health System; Public Health.
\end{abstract}

\title{
INTRODUÇÃO
}

A criação do Sistema Único de Saúde (SUS) foi sem dúvida o maior movimento de inclusão social já presenciado na história do Brasil, e em termos constitucionais representou a afirmação política de compromisso do Estado para com o direito de seus cidadãos (BRASIL, 2007). Foi criado a partir das disposições constitucionais de 1988 e implementado por leis promulgadas em 1990, representando assim o principal comprador de serviços de saúde no país e compondo um sistema misto com os serviços contratados por operadoras de seguros privados para seus beneficiários e aqueles pagos por desembolso direto (RIBEIRO, 2009).

Sendo assim, o SUS assume um papel fundamental no quadro sanitário brasileiro, tanto pela estrutura de organização institucional da área da saúde e modelo de atendimento à clientela como pela mudança impressa nas formas de direcionar, conceber, pensar e fazer a assistência à saúde no país (BRASIL, 1991). E o sistema de saúde brasileiro vive um 
Raquel Cristina Muniz de Souza, Cledir Lago, Aline da Rosa Paganini, Eduarda Nichele, Fernanda Januário Batista, Luana Dal Pont, Sabrina Furtunato de Ávila, Francielle Rosso Mazzuchello, Carla Maragno, Ivanir Prá da Silva Thomé, Luiz Gustavo Martins, Joni Marcio de Farias, Priscyla Waleska Simões, Lisiane Tuon

momento onde passa por intensos avanços, principalmente no que se refere à oferta de diversos programas, projetos e políticas, cujos resultados refletem em inegáveis benefícios para a população brasileira (SOUZA; COSTA, 2009a).

Apesar desses avanços e conquistas, o SUS apresenta também alguns desafios a serem superados, como problemas de implementação, implantação, financiamento e gestão (SOUZA; COSTA, 2009b). Em relação à atuação de trabalhadores qualificados na área da saúde, a formação e o preparo de profissionais em suas respectivas instituições de ensino tem profunda associação com a atuação nas instâncias do SUS, já que muitas instituições de ensino ainda privilegiam o modelo assistencial (LIRA NETO et al., 2013). É muito presente ainda o fato de que profissionais da saúde ainda encontram grandes dificuldades em trabalhar de forma integrada, onde impera a cultura da fragmentação do cuidado, cujo objetivo volta-se para o oferecimento da maior quantidade possível de serviços de saúde, centrados na consulta médica, voltada a tratar as enfermidades, por meio da clínica e com a intermediação crescente de tecnologias (CANESQUI, 1995).

O Programa denominado Vivências e Experiências na Realidade do SUS da Região Carbonífera de SC (Viver SUS), realizado pela Universidade do Extremo Sul Catarinense, surgiu com essa proposta de provocar uma mudança de consciência na forma de atuar na assistência à população. Nesse sentido o III VIVER SUS UNESC e I VER SUS UNESC na Região Carbonífera de Santa Catarina oportuniza o convívio, a discussão da organização do sistema de saúde brasileiro e a aprendizagem crítica contemplando estudantes de diversos cursos da área da saúde (CADERNO DIDÁTICA VIVER-SUS, 2014).

O objetivo geral do Viver SUS é oferecer aos estudantes a oportunidade de conhecer e refletir sobre o SUS, assim como promover discussões e reflexões com os trabalhadores de saúde que atuam no sistema sobre o fortalecimento da atenção em saúde coletiva oferecida à população. O projeto conta ainda com objetivos específicos de valorizar e potencializar o compromisso ético-político dos participantes do espaço de vivência no processo da Reforma Sanitária, provocar reflexões acerca do papel do estudante enquanto agente transformador da realidade social, contribuir para a construção da consciência acerca da saúde em seu conceito ampliado, sensibilizar gestores, trabalhadores e formadores do sistema de saúde, contribuir para o amadurecimento da prática interdisciplinar e multiprofissional, contribuir para o debate sobre o projeto político-pedagógico da graduação e sobre a implementação das diretrizes curriculares da saúde e estimular a inserção dos estudantes no Movimento Estudantil e em outros movimentos sociais. 
Diante dos objetivos propostos pelo projeto, torna-se evidente sua relevância como objeto de pesquisa e como ferramenta para promoção da integração dos futuros profissionais à realidade da organização dos serviços, a partir do conhecimento dos aspectos de gestão do sistema, estratégias de atenção, controle social e os processos de educação na saúde.

Assim, o estudo tem como objetivo relatar a experiência e a vivência dos residentes do programa de Residência Multiprofissional em Saúde Coletiva e Saúde da Família, e dos acadêmicos dos cursos de Farmácia, Educação Física, Psicologia, Biomedicina e Nutrição da UNESC no projeto Ver Viver SUS.

\section{MÉTODO}

Relato de experiência, vivenciado pelos residentes do Programa de Residência Multiprofissional em Saúde Coletiva/Saúde da Família das áreas de Enfermagem e Educação Física, juntamente com os acadêmicos dos cursos de Farmácia, Educação Física, Nutrição, Biomedicina e Psicologia. E tutores docentes em Farmácia, Enfermagem e Odontologia, totalizando 11 participantes.

Este relato faz parte do programa denominado Vivência e Experiências na realidade do SUS da região carbonífera de Santa Catarina. A experiência e vivência no SUS ocorreu durante o recesso escolar da Universidade do Extremo Sul Catarinense - UNESC entre os dias 21 a 25 de julho do ano de 2014 no município de Balneário Rincão, onde os residentes ficaram imersos durante este período no município.

A metodologia adotada foi a pesquisa de campo do tipo exploratória e descritiva, e teve como ferramenta de coleta de dados, diário de campo, rodas de discussões, registros por meio de fotos e entrevistas com o gestor de saúde. O cenário do Projeto VIVER SUS UNESC foi a rede de serviços de saúde do município.

A seleção dos estudantes foi realizada na Unidade Acadêmica da Saúde (UNASAU) por meio de uma carta de intenção na qual os acadêmicos deveriam especificar os motivos pelos quais gostariam de vivenciar esta experiência. Dentre os critérios de inclusão destacamse: dispor de tempo integral durante a realização do projeto e estar devidamente matriculado e frequentando cursos pertencentes ou relacionados à área da saúde. Com base nesses critérios foram selecionados cinco (5) acadêmicos para a cidade de Balneário Rincão - SC.

Todos os estudantes selecionados tiveram a oportunidade de conhecer diferentes instâncias em que o SUS atua, como Unidades Básicas de Saúde (UBS), Estratégia Saúde da Família (ESF), Conselhos Municipais de Saúde, Academia da Saúde, Serviços Especializados, Vigilância Epidemiológica, Vigilância Sanitária e do Meio Ambiente, 
Raquel Cristina Muniz de Souza, Cledir Lago, Aline da Rosa Paganini, Eduarda Nichele, Fernanda Januário Batista, Luana Dal Pont, Sabrina Furtunato de Ávila, Francielle Rosso Mazzuchello, Carla Maragno, Ivanir Prá da Silva Thomé, Luiz Gustavo Martins, Joni Marcio de Farias, Priscyla Waleska Simões, Lisiane Tuon

Farmácia Municipal, Prefeitura e Secretaria de Saúde, além de participar de reuniões com as comunidades locais, profissionais de saúde, conselheiros e grupos de educação em saúde. Também foi visitada uma Clínica Particular e uma Organização Não-Governamental (ONG) que trata de dependentes químicos, do sexo masculino. Após cada dia de estágios e vivência no SUS, os participantes debatiam as situações vistas em forma de sistematizações e elaboravam relatórios diários, destacando pontos importantes como as potencialidades, as situações limites e as possíveis intervenções a serem traçadas para cada local/situação. Os acadêmicos também tinham como base para as discussões o uso de textos disponibilizados a todos os participantes por meio de CD-ROM, palestras assistidas durante o processo e apoio dos residentes que atuaram como facilitadores do processo.

\section{RESULTADOS}

Durante o período do Viver SUS, os facilitadores e os acadêmicos puderam ter um contato muito próximo com a atenção básica em saúde realizada no município do Balneário Rincão. Essa proximidade com os serviços permitiu à equipe conhecer melhor as potencialidades e as fragilidades do serviço. Os resultados dessa vivência estão descritos a seguir.

Durante as visitas a algumas Estratégia Saúde da Família (ESF) constatou-se que os locais onde profissionais trabalhavam são bem estruturados, com estruturas físicas novas e dispunham de um lugar específico para realizarem educação em saúde com a população, seja na própria unidade, seja em centros comunitários e praças com amplo espaço para realização de tal atividade. Esse cenário possibilita que o diálogo educativo entre os profissionais e a população não se torne um mero discurso e seja acompanhado de um movimento de fortalecimento, empowerment (empoderamento), econômico, político, social e cultural dos indivíduos e grupos socialmente subordinados (LEFÈVRE; LEFÈVRE, 2004), o que leva ao fortalecimento de espaços como estes.

Educação em Saúde é um campo de práticas que se dão no nível das relações sociais normalmente estabelecidas pelos profissionais de saúde, entre si, com a instituição e, sobretudo, com o usuário, no desenvolvimento cotidiano de suas atividades (L'ABATTE, 1994). Diante disso, a realização de grupos terapêuticos nas Estratégias de Saúde da Família vem se tornando de suma importância cada vez mais, proporcionando troca de experiências tanto para profissionais quanto para usuários, conforme observado nos grupos ao qual a equipe do Viver SUS teve a oportunidade de participar e trouxe contribuições de suas respectivas áreas do conhecimento. 
Antigamente, os Grupos de Educação em Saúde não tinham boa aceitação pelos pacientes, pois ambos não tinham conhecimento da importância dos cuidados com sua saúde e práticas que podem melhorar sua qualidade de vida. Com a educação em saúde, são recrutadas ideias dinâmicas que envolvem os usuários do grupo despertarem da conscientização para a prevenção e promoção de saúde.

Foi realizado um Grupo Operativo Terapêutico debatendo o tema Saúde e Qualidade de Vida, atividade educativa na sala de espera, Oficina de Planejamento Estratégico e debate sobre o papel da Atenção Básica com os profissionais de saúde e um encontro com o controle social, além de uma sensibilização entre os profissionais de saúde em conjunto com as lideranças da comunidade de um bairro.

A assistência domiciliar à saúde é uma categoria da atenção domiciliar à saúde que pode ser também denominada atendimento ou cuidado domiciliar e baseia-se na plena interação do profissional com o paciente, sua família e com o cuidador, quando esse existe. Ela constitui um conjunto de atividades de caráter ambulatorial, programadas e continuadas, desenvolvidas em domicílio, e pode ser instrumentalizada pela visita ou internação domiciliar (MOZARA; LACERDA, 2006). Essa forma de assistência pôde ser observada nos bairros do município a qual foi realizada por intermédio da Enfermeira da Estratégia de Saúde da Família em conjunto com as Agentes Comunitárias de Saúde (ACS) e com o Fisioterapeuta. Foram realizadas duas visitas domiciliares permitindo às acadêmicas um contato direto com a realidade do território e do contexto familiar daquela ESF.

Para fechamento das atividades foi realizada a feira de saúde, que ocorreu no período matutino na Academia de Saúde do município, sendo aberta para todos os usuários do sistema. Foram oferecidos os serviços de aferição de Pressão Arterial, testes rápidos de HIV, sífilis e hepatite $\mathrm{C}$, testes de estresse e depressão, avaliação antropométrica e avaliação bucal. Essas ações se constituem não apenas em uma extensão dos conhecimentos acadêmicos adquiridos na Universidade, mas principalmente perceber a importância da prática e da interação com os indivíduos que recebem o serviço.

O bom relacionamento e a interação entre os diversos profissionais da área da saúde, entre a universidade e as famílias atendidas, auxiliam na aceitação das pessoas para o acompanhamento frequente e a busca da satisfação das necessidades de saúde. Quando todos os membros conhecem as necessidades das famílias, a abordagem acontece em sua totalidade e é mais eficaz, pois toda a equipe participa do acompanhamento (OLIVEIRA, 2006). 
Raquel Cristina Muniz de Souza, Cledir Lago, Aline da Rosa Paganini, Eduarda Nichele, Fernanda Januário Batista, Luana Dal Pont, Sabrina Furtunato de Ávila, Francielle Rosso Mazzuchello, Carla Maragno, Ivanir Prá da Silva Thomé, Luiz Gustavo Martins, Joni Marcio de Farias, Priscyla Waleska Simões, Lisiane Tuon

\section{CONCLUSÃO}

O projeto Viver SUS se configura como um dispositivo agregador de conhecimento sobre o sistema de saúde brasileiro que contribui em muito para a formação profissional dos acadêmicos. Esse conhecimento, adquirido a partir das vivências, de certa forma provoca uma mudança na percepção em relação ao serviço público e consequentemente estimula os participantes a se envolver com os serviços, com a população e com os profissionais.

Essa experiência possibilitou a aprendizagem prática não adquirida na academia, pois permitiu que os acadêmicos desenvolvessem atividades de educação em saúde com a população e percebessem a importância e o impacto que esse diálogo educativo exerce nos usuários.

Outra dimensão do projeto observada diz respeito aos desafios e possibilidades que o SUS oferece nas práticas diárias, mediante a diversidade cultural das populações. Devemos estar preparados para usar a criatividade e a agilidade humana de criar e recriar as diversas situações que a saúde pública nos apresenta.

É importante destacar ainda a relevância do contato que os participantes têm com os gestores de saúde. A experiência desses gestores contribui para a geração de uma consciência crítica por parte de acadêmicos e facilitadores, quando estes podem visualizar melhor as fragilidades e potencialidades dos serviços oferecidos no município. Esse contato, aliado à interação com os profissionais pertencentes às equipes de saúde do Balneário Rincão, oportunizou a compreensão da importância que a interdisciplinaridade, intersetorialidade e multiprofissionalidade têm no serviço de saúde. O trabalho dentro da equipe se torna também uma reprodução desse contexto, onde o contato com os profissionais da Residência Multiprofissional permite adquirir experiências e troca de ideias de grande valia para a formação acadêmica e profissional.

\section{REFERÊNCIAS}

BRASIL. CONSELHO NACIONAL DE SECRETÁRIOS DE SAÚDE. Sistema Único de Saúde. Brasília, 2007.

BRASIL. CONGRESSO NACIONAL. Constituição da República Federativa do Brasil. Promulgada em 05 de outubro de 1988. São Paulo: Editora Atlas, 1991.

BRASIL, Ministério da Saúde (MS). Portaria No 2.488, de 21 de Outubro de 2011: Aprova a Política Nacional de Atenção Básica, estabelecendo a revisão de diretrizes e normas para a organização da Atenção Básica, para a Estratégia Saúde da Família (ESF) e o Programa de Agentes Comunitários de Saúde (PACS). Brasília: Ministério da Saúde, 2011. 
L'ABBATE, Solange. Educação em saúde: uma nova abordagem. Cadernos de Saúde Pública, v. 10, n. 4, p. 481-490, 1994.

LEFEVRE, Fernando; LEFEVRE, Ana Maria Cavalcanti. Promoção de Saúde. 2004.

LIRA NETO, José Cláudio Garcia et al. VER-SUS: UM RELATO DE EXPERIÊNCIA SOBRE UMA VIVÊNCIA-ESTÁGIO NA REALIDADE DO SISTEMA ÚNICO DE SAÚDE. Rev Enferm, Recife, v.7, p.1042-1046, 2013.

GIACOMOZZI, Clélia Mozara; LACERDA, Maria Ribeiro. A prática da assistência domiciliar dos profissionais da estratégia de saúde da família. Texto contexto enferm., v. 15, n. 4, p. 645-53, 2006.

MANCIA, Joel Rolim; CABRAL, Leila Chaves; KOERICH, Magda Santos. Educação permanente no contexto da enfermagem e na saúde. Rev. Bras. Enferm., v. 57, n. 5, p. 605-10, 2004.

CANESQUI, Ana Maria. Dilemas e desafios das ciências sociais na saúde coletiva. In: Saúde em debate. Hucitec, 1995.

DE OLIVEIRA, Elaine Machado; SPIRI, Wilza Carla. Programa Saúde da Família: a experiência de equipe multiprofissional. Rev Saúde Pública, v. 40, n. 4, p. 727-33, 2006.

SOUZA, Georgia Costa de Araújo; COSTA, Iris do Céu Clara. O SUS nos seus 20 anos: reflexões num contexto de mudanças. Saúde Soc. São Paulo, São Paulo, v. 19, n. 3, p.509-517, 20 jul. 2009. 